\section{Comparison of breast tissue markers for tumor localization in breast cancer patients undergoing neoadjuvant chemotherapy}

Ja Ho Koo, Eun-Kyung Kim, Hee Jung Moon, Jung Hyun Yoon, Vivian Youngjean Park, Min Jung Kim

Department of Radiology, Severance Hospital, Yonsei University College of Medicine, Seoul, Korea

Purpose: The purpose of this study was to compare the visibility of breast tissue markers in cases of breast cancer on ultrasonography (US) after neoadjuvant chemotherapy (NAC) and to analyze whether the type of marker affected the choice of localization method after NAC.

Methods: We included 153 tissue markers inserted within breast cancers that showed pathologically complete response ( $p C R$ ) after NAC from January 2012 to April 2017. One of three types of markers (a surgical clip, Cormark, or UltraClip) was inserted. Medical records and imaging findings were retrospectively reviewed. We compared the visibility of the different types of tissue markers on US after NAC, and also compared the imaging modalities used in the preoperative localization. The chi-square test, Fisher exact test, and multiple logistic regression were used for analysis.

Results: Of the 153 tissue markers, 56 were surgical clips, 61 Cormark, and 36 UltraClip. After $N A C$, residual lesions were not seen on US in 42 cases (27.5\%). In multivariate analysis, the visibility of the surgical clips and Cormark markers was better than that of the UltraClip markers (odds ratio [OR], 5.467; 95\% confidence interal [CI], 1.717 to 17.410; $\mathrm{P}=0.004$ and OR, 3.045; $95 \% \mathrm{Cl}, 1.074$ to $8.628 ; \mathrm{P}=0.036$, respectively). Among the 131 cases where localization targeting the marker was required, the proportion of US-guided localizations was significantly higher when a surgical clip was used than when an UltraClip marker was used (OR, 5.566; 95\% $\mathrm{Cl}, 1.610$ to $19.246 ; \mathrm{P}=0.007)$ in the multivariate analysis.

Conclusion: The type of breast tissue marker affected its visibility on US in cases with $p C R$ after NAC, which in turn affected the localization methodology.

Keywords: Breast; Ultrasonography; Localization; Surgical clip

\section{Introduction}

Neoadjuvant chemotherapy (NAC) is widely considered in breast cancer patients who are potential candidates for adjuvant chemotherapy [1]. NAC has been proven to increase overall survival and
ULTRA

SONO

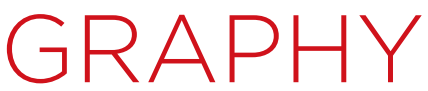

ORIGINAL ARTICLE

https://doi.org/10.14366/usg. 19004 pISSN: 2288-5919 • elSSN: 2288-5943 Ultrasonography 2019;38:336-344

Received: January 8, 2019

Revised: March 22, 2019

Accepted: April 7, 2019

Correspondence to:

Min Jung Kim, MD, PhD, Department of Radiology, Severance Hospital, Yonsei University College of Medicine, 50-1 Yonsei-ro, Seodaemun-gu, Seoul 03722, Korea

Tel. $+82-2-2228-7400$

Fax. +82-2-2227-8337

E-mail: mines@yuhs.ac

This is an Open Access article distributed under the terms of the Creative Commons Attribution NonCommercial License (http://creativecommons.org/ licenses/by-nc/4.0/) which permits unrestricted noncommercial use, distribution, and reproduction in any medium, provided the original work is properly cited.

Copyright (C) 2019 Korean Society of Ultrasound in Medicine (KSUM)

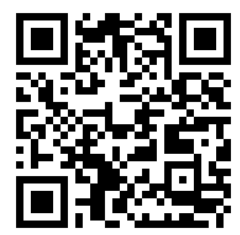

How to cite this article:

Koo JH, Kim EK, Moon HJ, Yoon JH, Park VY Kim MJ. Comparison of breast tissue markers for tumor localization in breast cancer patients undergoing neoadjuvant chemotherapy. Ultrasonography. 2019 0ct;38(4):336-344. 
disease-free survival, while providing an increased chance of being able to perform breast-conserving surgery (BCS) [2]. The safety and efficacy of breast tissue marker insertion before NAC are now also commonly accepted. Markers are used to demarcate the biopsy area because a residual tumor after NAC may be clinically occult and further surgery may be required pending a margin status review by pathology. The placement of these markers is more accurately done under ultrasound (US) guidance than under stereotactic guidance $[3,4]$.

When BCS is considered, needle localization is necessary for marker insertion prior to surgery. A marker may be clearly visible on US if it is present within a hypoechoic lesion, and US-guided localization is preferred because US-guided biopsy has several advantages over stereotactic biopsy [5]. However, in patients with pathologically complete response $(p C R)$, the lesions might have dramatically shrunk to the point of not being visible on US. Wellvisible breast tissue markers need to be placed in these lesions in order to enable better surgical options and outcomes $[3,6]$.

While Fajardo et al. [7] introduced the benefits of the microcoil as a breast marker, Thomassin-Naggara et al. [8] introduced surgical clips inserted with an 18-gauge spinal needle. Before many types of commercial breast tissue markers were available for patients undergoing NAC, surgical clips were used as breast markers. Breast tissue markers can be categorized into commercial metallic markers and markers with surrounding material. Commercial metallic markers are simply made of 2- to 3-mm pieces of titanium or stainless steel, and are the most commonly used markers today. Markers with surrounding material were developed for long-term visibility, and the embedding materials can be collagen, polylactic acid, polyglycolic acid or starch pellets, or hydrogel. These markers have several advantages. First, they fill the space cavity, thereby decreasing the risk of marker migration. Second, the markers have a hemostatic effect because they place direct pressure on adjacent tissue. Third, they enhance visibility on US. However, a previous study reported that although markers with a microfiber pad were clearly seen on US just after insertion, visibility greatly decreased after 6-8 weeks, making them ineffective as breast markers for patients who have undergone NAC [8]. The effectiveness of surgical clip placement has been studied before for breast cancer, but insufficient studies have compared different types of breast tissue markers after NAC [9]. It is necessary to investigate which types of breast tissue markers are more visible on specific imaging modalities, because such knowledge would help radiologists determine the most suitable type of breast tissue marker for each patient before insertion.

The objective of this study was to compare the visibility of breast tissue markers after NAC on US and to analyze whether the type of breast tissue marker affected the choice of localization method.

\section{Materials and Methods}

This retrospective study was approved by the Institutional Review Board of our institution with a waiver of informed consent.

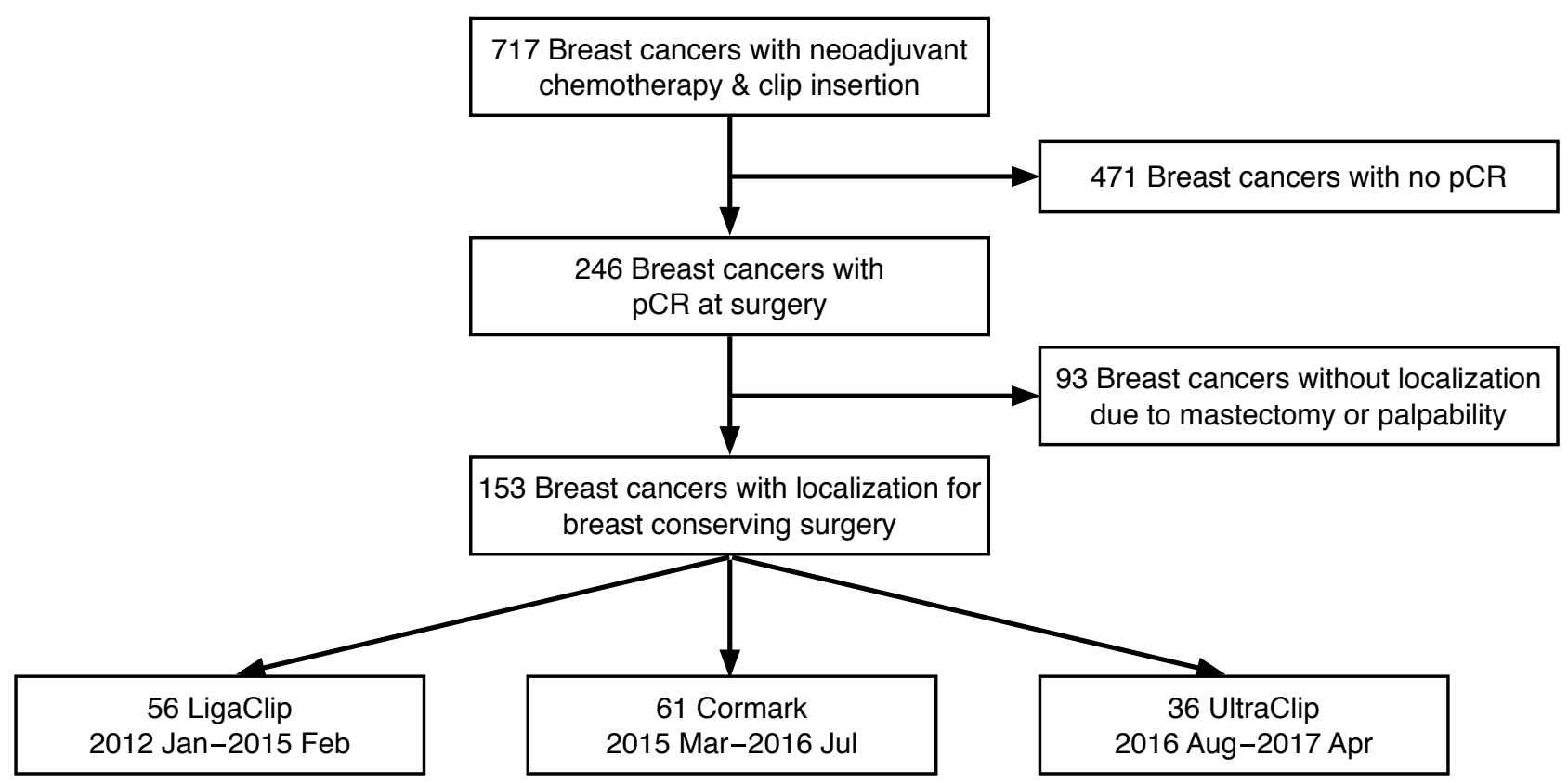

Fig. 1. Selection process for the study population. $p C R$, pathologic complete response. 


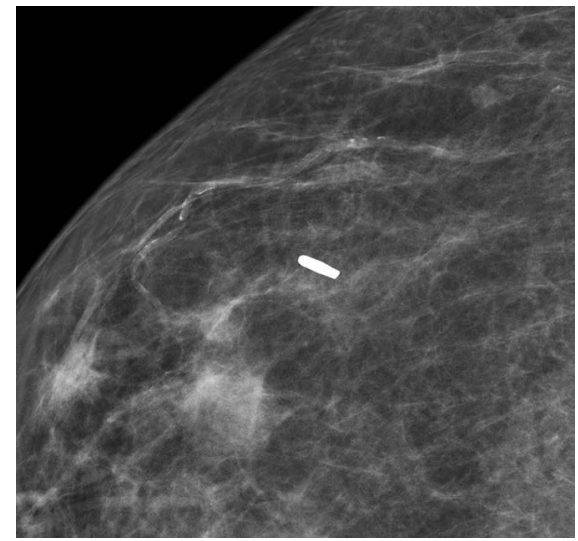

A

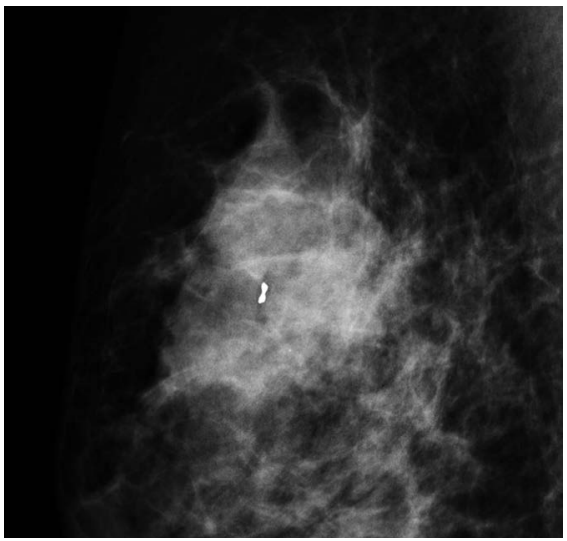

$B$

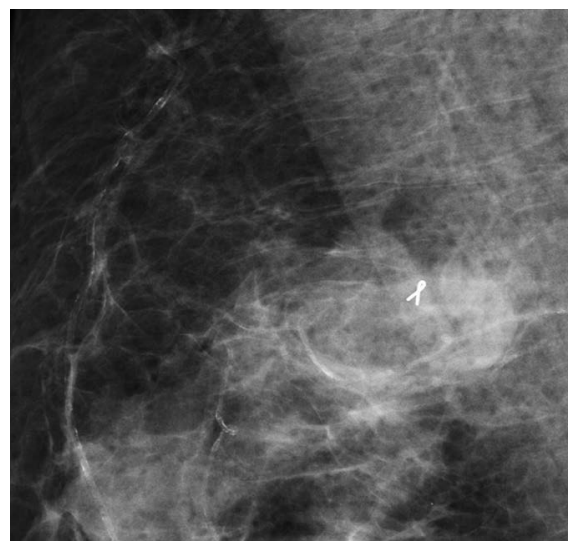

C

Fig. 2. Mammograms of three markers: LigaClip (A), Cormark (B), and UltraClip (C).

Table 1. Comparison of clinicopathologic data for the patients who underwent breast tissue marker insertion

\begin{tabular}{|c|c|c|c|c|}
\hline Parameter & LigaClip $(n=56)$ & UltraClip $(n=36)$ & Cormark $(n=61)$ & P-value \\
\hline Age, median (interquartile range, yr) & $52(45.0-57.0)$ & $47.5(40.5-52.75)$ & $51(41.5-57.0)$ & $0.143^{2 \mathrm{a}}$ \\
\hline \multicolumn{5}{|l|}{ Pathology } \\
\hline Luminal A & 3 & 2 & 4 & $0.565^{b}$ \\
\hline Luminal B & 6 & 5 & 10 & \\
\hline $\mathrm{HER}^{+}$ & 14 & 10 & 16 & \\
\hline TNBC & 33 & 19 & 31 & \\
\hline pCR with DCIS & 18 & 12 & 12 & $0.229^{c}$ \\
\hline pCR without DCIS & 38 & 24 & 49 & \\
\hline \multicolumn{5}{|l|}{ Dense breast type } \\
\hline A & 1 & 0 & 0 & $0.408^{b}$ \\
\hline B & 9 & 4 & 9 & \\
\hline C & 43 & 29 & 42 & \\
\hline D & 3 & 3 & 10 & \\
\hline
\end{tabular}

HER, human epidermal growth factor receptor 2; TNBC, triple-negative breast cancer; $\mathrm{PCR}$, pathologic complete response; DCIS, ductal carcinoma in situ.

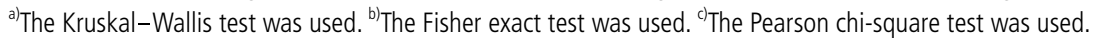

\section{Patients}

From January 2012 to April 2017, we inserted breast tissue markers before NAC for 717 breast cancers that were biopsied under US guidance. Among them, 246 cases were confirmed to show $p C R$ at surgery. While 153 of the $p C R$ cases underwent BCS after localization, the remaining 93 underwent total mastectomy. The final study population consisted of patients confirmed to have achieved pCR who were treated by BCS (Fig. 1). pCR was defined as the absence of invasive cancer in both the breast and lymph nodes with or without the presence of ductal carcinoma in situ (DCIS) [1]. We retrospectively reviewed the patients' medical records and recorded which of the three types of breast tissue marker was inserted: a surgical clip (LigaClip MCA MSM20, Ethicon Endo-
Surgery, Somerville, NJ, USA), an UltraClip marker (C. R. Bard Inc., Murray Hill, NJ, USA), or a Cormark marker (Ethicon Endo-Surgery Inc, Cincinnati, OH, USA) (Fig. 2). From January 2012 to February 2015, LigaClip markers were inserted, and from March 2015 to July 2016, Cormark markers were inserted. After August 2016, UltraClip markers were inserted. The clinicopathologic data for the three groups are given in Table 1.

\section{Breast Tissue Marker Implantation}

Markers were implanted just before NAC by one of 13 radiologists who subspecialized in breast imaging (Fig. 3). After local anesthesia was applied under aseptic dressing, a 14-gauge coaxial guiding needle was inserted into the breast cancer under US guidance. The 
inner stylet was pulled off and the breast tissue marker (LigaClip or Cormark) was inserted through an introducer. The inner stylet was then reinserted in order to insert the marker completely into the lesion. UltraClip markers were inserted without a coaxial guiding needle. We inserted one marker into each cancer lesion. US was used to confirm the location of the marker immediately after the implantation procedure. Mammography was also used to confirm the location of the marker.

\section{Imaging Protocol and Assessment}

Ultrasonography and mammography were used to evaluate breast cancer lesions just before starting NAC and before surgery. In the 148 patients, 153 lesions were identified on US just before
NAC, and breast tissue markers were inserted into each lesion. The insertion of the marker was also confirmed by mammography. After each chemotherapy cycle was finished, the visibility of the tumor lesion and breast tissue markers was evaluated on both US and mammography, and these findings were recorded in the imaging reading reports. We considered a marker to be visible if it was indicated as visible in the imaging reading reports. All US examinations were performed by radiologists who subspecialized in breast imaging. The echogenicity of the surrounding background (hypoechoic, isoechoic, or hyperechoic) and marker depth were also analyzed. In cases where no marker was seen on US, echogenicity was measured in the presumed area and marker depth was measured on images taken immediately after marker insertion.

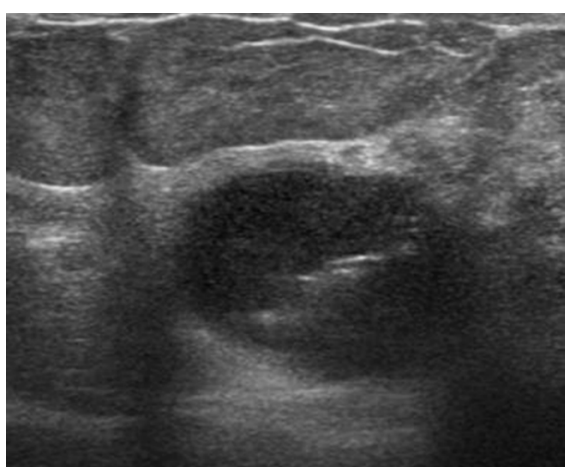

A

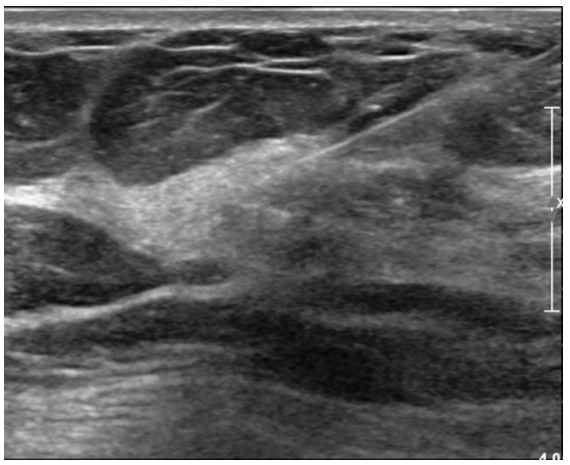

C

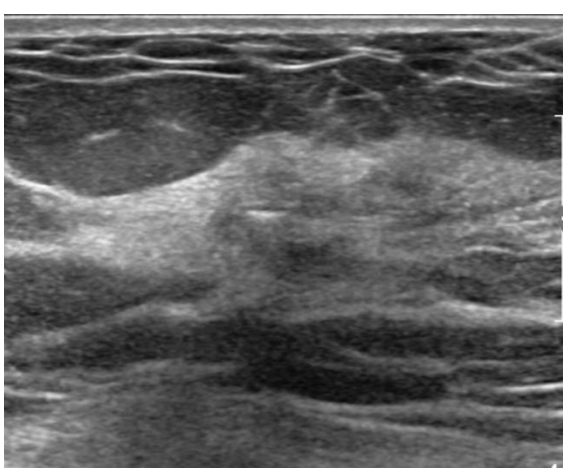

B

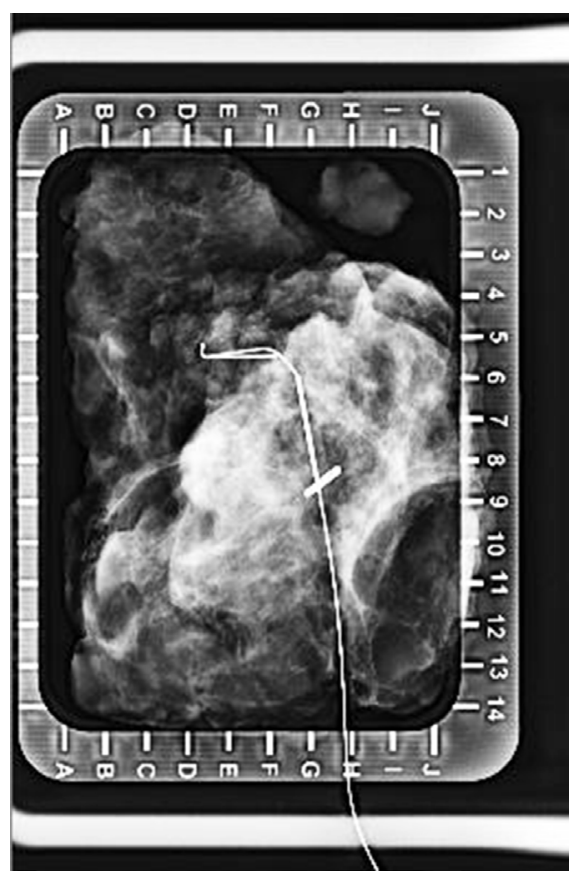

D
Fig. 3. Visible LigaClip with ultrasoundguided localization (no residual lesion) in a 50-year-old woman with triple-negative breast cancer and no ductal carcinoma in situ.

A. Initial tumor lesion was seen on ultrasound with LigaClip. B. Only the LigaClip is visible on ultrasound after neoadjuvant chemotherapy. C. The localization needle is inserted into the lesion. D. Localization was confirmed using specimen mammogram. 
The tumor lesions were localized before surgery. When the marker was clearly visible on US, it was localized under US guidance, and if the marker was not visible on US or if microcalcifications needed to be targeted through a mammogram, it was localized under mammography guidance. After surgery, we confirmed that the localization needle and markers had been removed through specimen mammograms.

\section{Data Analysis}

We retrospectively reviewed patients' medical records to evaluate whether the breast tissue markers were visible on US or mammography. Localization methods in partial mastectomy patients were reviewed. Additionally, the surgical method and pathology reports from surgical specimens were reviewed. Estrogen receptor, progesterone receptor, human epidermal growth factor receptor 2 (HER2), and Ki-67 were analyzed to categorize the pathologic type of breast cancer as triple-negative breast cancer (TNBC), luminal type $A$ and $B$, or HER2 ${ }^{+}$. The presence of DCIS was also reviewed.

Groups were compared regarding the visibility of the breast tissue marker on US after NAC and the methodology of localization using the chi-square test and the Fisher exact test. The relationships between marker visibility and the type of breast cancer pathology or presence of DCIS were also analyzed using the chi-square test and the Fisher exact test. We performed an analysis of all cases, then analyzed selected cases in which no residual lesion was seen on US. Furthermore, we conducted multiple logistic regression for the multivariate analysis. P-values less than 0.05 were considered to indicate statistical significance. SPSS version 23.0 (IBM Corp., Armonk, NY, USA) was used for statistical analysis. Patients were categorized by the type of breast tissue marker inserted.

\section{Results}

In 148 patients (median age, 51 years; range, 26 to 72 years) who underwent BCS after localization, three types of breast tissue markers were inserted in 153 breast lesions that demonstrated $p C R$ at surgery: 56 LigaClip markers, 61 Cormark markers, and 36 UltraClip markers. At the US evaluations after NAC, a residual tumor lesion was seen in 111 cases $(72.5 \%)$, but not in the remaining 42 cases (27.5\%). Markers were seen on US in 127 cases overall (83.0\%), and in 30 cases (71.4\%) with no residual lesion visible on US.

\section{Comparison of the Visibility of the Breast Tissue Marker by Type}

Marker visibility was significantly more common when using LigaClip $(91.1 \%, 51$ of 56$)$ or Cormark markers $(86.9 \%, 53$ of 61$)$ than when using UltraClip markers (63.9\%, 23 of $36 ; \mathrm{P}=0.002$ and $\mathrm{P}=0.008$, respectively) in all cases (Figs. $3-5$ ). In cases where no residual lesion was noted on US after NAC, marker visibility was significantly more common when using LigaClip markers $(88.2 \%, 15$ of 17) than when using UltraClip markers $(46.7 \%, 7$ of $15, \mathrm{P}=0.021)$. Marker visibility tended to be more common in cases using Cormark markers ( $80 \%$ [8 of 10]) than those using UltraClip markers (46.7\%, 7 of 15), but without statistical significance ( $P=0.211)$ (Table 2).

\section{Clinicopathologic Factors Affecting the Visibility of the Breast Tissue Marker on US}

Of the 153 breast lesions, nine were luminal A breast cancer, 21 were luminal $B$ breast cancer, 83 were TNBC, and 40 were HER2 ${ }^{+}$ breast cancer. There were 42 cases with a residual DCIS component, and the remaining 111 did not have DCIS or an invasive component. The presence of residual DCIS and the molecular type of breast cancer did not affect the visibility of the breast tissue marker on US after NAC ( $P=0.845$ and $P=0.583$, respectively).

\section{US Echogenicity and Marker Depth}

Among the 153 cases, the surrounding background was categorized as hypoechoic in 67 cases and as isoechoic in 86 cases. Echogenicity

Table 2. Comparison of visibility on ultrasound between breast tissue markers

\begin{tabular}{|c|c|c|c|c|c|c|}
\hline & \multicolumn{2}{|c|}{ LigaClip } & \multicolumn{2}{|c|}{ UltraClip } & \multicolumn{2}{|c|}{ Cormark } \\
\hline & Yes & No & Yes & No & Yes & No \\
\hline All cases $(n=153)$ & \multicolumn{2}{|c|}{56} & \multicolumn{2}{|c|}{36} & \multicolumn{2}{|c|}{61} \\
\hline No. (\%) & $51(91.1)$ & $5(8.9)$ & $23(63.9)$ & $13(36.1)$ & $53(86.9)$ & $8(13.1)$ \\
\hline P-value & & $0.002^{\mathrm{a})}$ & & & $0.008^{b)}$ & \\
\hline No residual lesion on ultrasound $(n=42)$ & \multicolumn{2}{|c|}{17} & \multicolumn{2}{|c|}{15} & \multicolumn{2}{|c|}{10} \\
\hline No. (\%) & $15(88.2)$ & $2(11.8)$ & $7(46.7)$ & $8(53.3)$ & $8(80.0)$ & $2(20.0)$ \\
\hline P-value & & $0.021^{\mathrm{a})}$ & & & $0.211^{b)}$ & \\
\hline
\end{tabular}

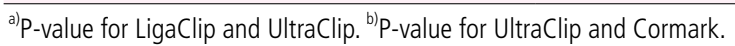




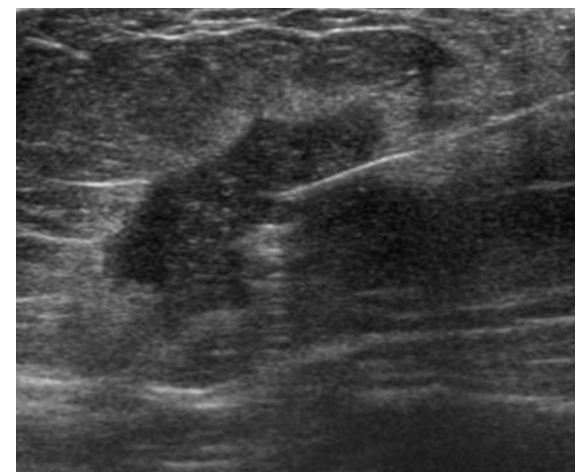

A

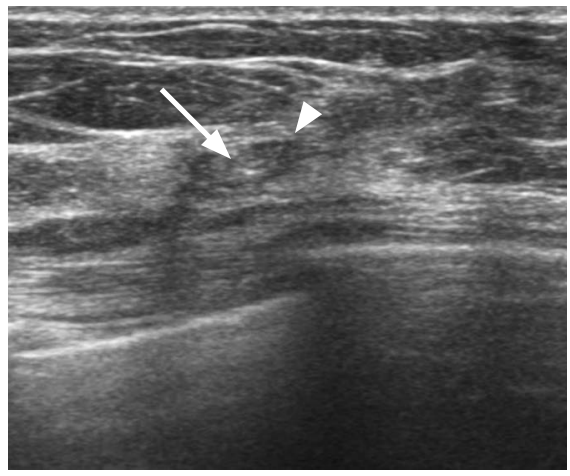

B

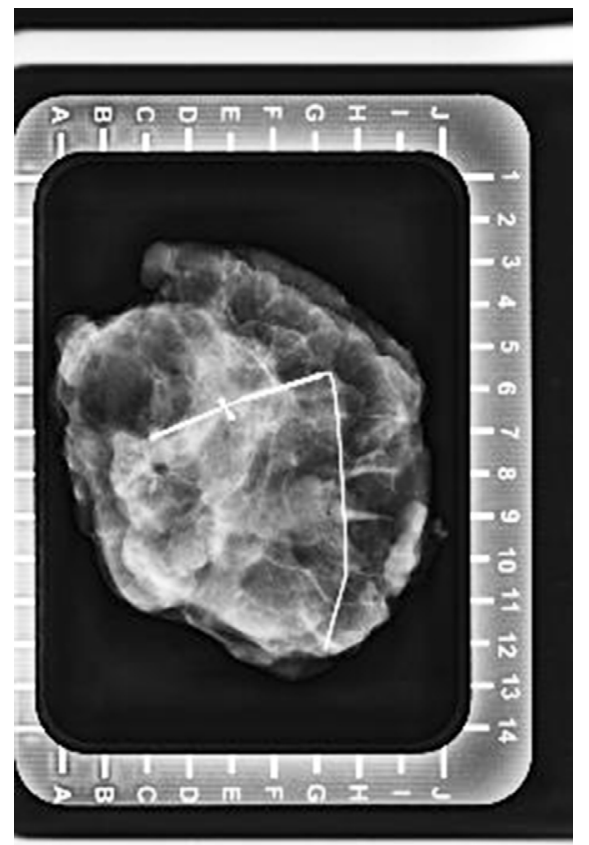

Fig. 4. Visible Cormark with ultrasound-guided localization (no residual carcinoma, fibrosis with collagen-like material deposition and giant cell reaction) in a 46-year-old woman with human epidermal growth factor receptor 2-positive breast cancer with residual ductal carcinoma in situ.

A. Initial tumor lesion was seen on ultrasound with the marker. B. Only the Cormark (marker itself [arrow], adjacent collagen [arrowhead]) is visible on ultrasound after neoadjuvant chemotherapy. C. Localization was confirmed using specimen mammogram.

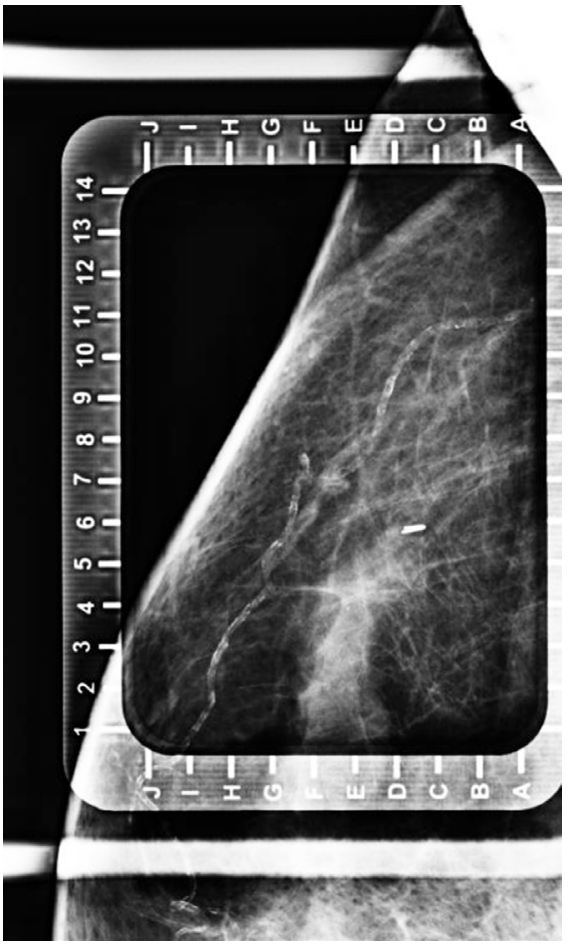

A

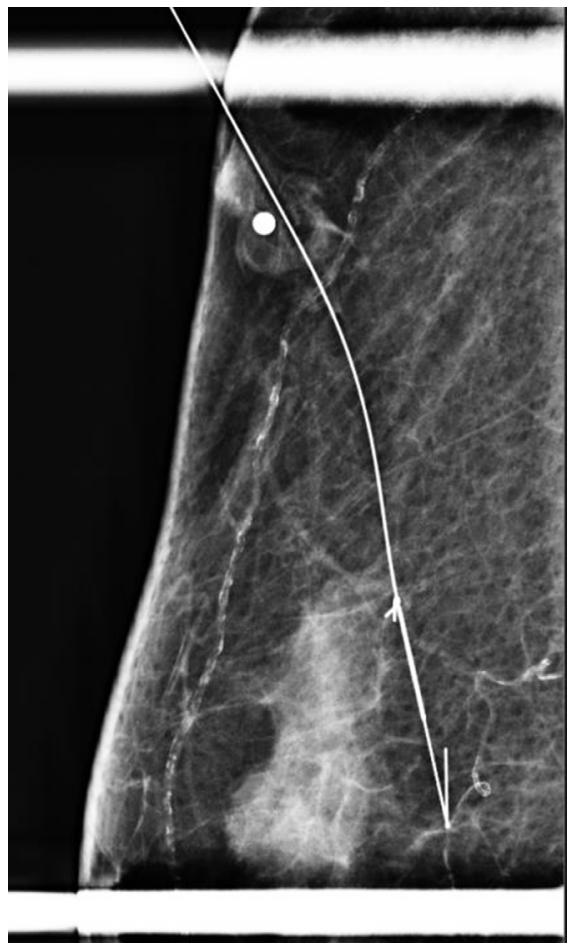

B

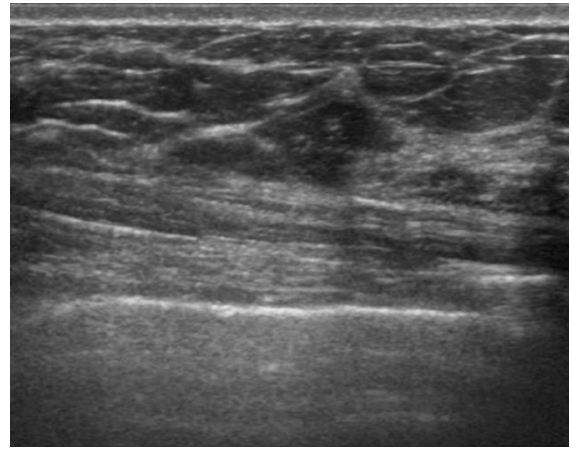

C

Fig. 5. Mammogram-guided localization due to the absence of a visible breast tissue marker (UltraClip) on ultrasonography (US) in a 64-year-old woman with human epidermal growth factor receptor 2-positive breast cancer with no ductal carcinoma in situ. A. The marker is visible on mammography. B. A guided wire is located in the marker. C. No residual lesion or marker is visible on US after neoadjuvant chemotherapy. 
did not affect visibility $(P=0.891)$. The marker depth was $11.7 \pm 3.78$ $\mathrm{mm}$ (mean \pm standard deviation), and also did not affect visibility $(\mathrm{P}=0.084)$.

\section{Comparison of Localization Methodology by Type of Breast Tissue Marker}

Of the 153 breast lesions, 131 were analyzed to determine whether the type of breast tissue marker affected localization methodology. Other lesions ( $n=16$ ) were localized by targeting microcalcifications on mammography (Fig. 5), and six cases were localized by targeting the tumor lesion, not the marker on US, because the marker was not visible on US. The marker was not targeted in these 22 cases, so they were excluded from this analysis. The percentage of US-guided localizations was significantly higher when using LigaClip markers $(90.4 \%, 47$ of 52$)$ or Cormark markers $(86.3 \%, 44$ of 51$)$ than when using UltraClip markers $(60.7 \%, 17$ of $28 ; P=0.003$ and $P=0.013$, respectively) in all cases. The proportion of US-guided localizations was significantly higher when using LigaClip markers $(81.3 \%, 13$ of 16) than when using UltraClip markers $(38.5 \%, 5$ of 13$)$ in cases where no residual lesion was noted on US after NAC ( $P=0.027)$. The proportion of US-guided localizations was higher when using Cormark markers $(75.0 \%, 6$ of 8$)$ than when using UltraClip markers $(38.5 \%, 5$ of 13$)$ when no residual lesion was noted on US after $N A C$, but this trend did not reach statistical significance $(P=0.183)$ (Table 3). The presence of DCIS and the molecular type of breast cancer did not affect the localization method after NAC $(P=0.657$ and $P=0.902$, respectively). The echogenicity of the surrounding background and marker depth did not affect the localization method after $\mathrm{NAC}(\mathrm{P}=0.397$ and $\mathrm{P}=0.084$, respectively).

\section{Multivariate Analysis}

We performed a multivariate analysis using multiple logistic regression (Tables 4, 5). In the univariate logistic regression analysis, the visibility of LigaClip or Cormark markers was better than that of UltraClip markers ( $P=0.003$ and $P=0.010$, respectively), and marker visibility was better in cases with US-visible residual

Table 3. Comparison of the localization methodology between breast tissue markers

\begin{tabular}{|c|c|c|c|c|c|c|}
\hline & \multicolumn{2}{|c|}{ LigaClip } & \multicolumn{2}{|c|}{ UltraClip } & \multicolumn{2}{|c|}{ Cormark } \\
\hline & US & MG & US & MG & US & MG \\
\hline All cases $(n=131)$ & \multicolumn{2}{|c|}{52} & \multicolumn{2}{|c|}{28} & \multicolumn{2}{|c|}{51} \\
\hline №. (\%) & $47(90.4)$ & $5(9.6)$ & $17(60.7)$ & $11(39.3)$ & $44(86.3)$ & $7(13.7)$ \\
\hline P-value & & $0.003^{\mathrm{a})}$ & & & $0.013^{b)}$ & \\
\hline No residual lesion on US ( $n=37$ ) & \multicolumn{2}{|c|}{16} & \multicolumn{2}{|c|}{13} & \multicolumn{2}{|c|}{8} \\
\hline No. (\%) & $13(81.3)$ & $3(18.8)$ & $5(38.5)$ & $8(61.5)$ & $6(75.0)$ & $2(25.0)$ \\
\hline P-value & & $0.027^{\mathrm{a})}$ & & & $0.183^{b)}$ & \\
\hline
\end{tabular}

US, ultrasound; MG, mammography.

a)P-value for LigaClip and UltraClip. ${ }^{\text {b) }}$-value for UltraClip and Cormark.

Table 4. Univariate and multivariate analysis for visibility on US between breast tissue markers

\begin{tabular}{|c|c|c|c|c|}
\hline \multirow{2}{*}{ Variable } & \multicolumn{2}{|c|}{ Univariate analysis } & \multicolumn{2}{|c|}{ Multivariate analysis } \\
\hline & Crude OR (95\% Cl) & P-value & Adjusted OR (95\% Cl) & P-value \\
\hline LigaClip vs. UltraClip & $5.765(1.839-18.078)$ & 0.003 & $5.467(1.717-17.410)$ & 0.004 \\
\hline Cormark vs. UltraClip & $3.745(1.367-10.256)$ & 0.010 & $3.045(1.074-8.628)$ & 0.036 \\
\hline Residual lesion visible on US & $2.771(1.158-6.635)$ & 0.022 & $2.430(0.956-6.177)$ & 0.062 \\
\hline Presence of DCIS & $1.033(0.399-2.670)$ & 0.947 & - & - \\
\hline Luminal A vs. TNBC & $0.406(0.091-1.819)$ & 0.239 & - & - \\
\hline Luminal B vs. TNBC & $1.217(0.315-4.699)$ & 0.775 & - & - \\
\hline $\mathrm{HER}^{+}$vs. TNBC & $1.150(0.406-3.256)$ & 0.793 & - & - \\
\hline Age & $1.029(0.986-1.073)$ & 0.195 & - & - \\
\hline Marker depth & $0.907(0.812-1.013)$ & 0.084 & - & - \\
\hline Hypoechoic than isoechoic & $0.891(0.382-2.079)$ & 0.790 & - & - \\
\hline
\end{tabular}

US, ultrasonography; OR, odds ratio; Cl, confidence interval; DCIS, ductal carcinoma in situ; TNBC, triple-negative breast cancer; HER2, human epidermal growth factor receptor 2. 
Table 5. Univariate and multivariate analysis for US-guided localization compared to mammogram-guided localization between breast tissue markers

\begin{tabular}{|c|c|c|c|c|}
\hline \multirow{2}{*}{ Variable } & \multicolumn{2}{|c|}{ Univariate analysis } & \multicolumn{2}{|c|}{ Multivariate analysis } \\
\hline & Crude OR (95\% Cl) & P-value & Adjusted OR $(95 \% \mathrm{Cl})$ & P-value \\
\hline LigaClip vs. UltraClip & $6.082(1.843-20.070)$ & 0.003 & $5.566(1.610-19.246)$ & 0.007 \\
\hline Cormark vs. UltraClip & $4.067(1.353-12.226)$ & 0.012 & $2.772(0.858-8.951)$ & 0.088 \\
\hline Residual lesion visible on US & $4.550(1.776-11.659)$ & 0.002 & $4.121(1.497-11.349)$ & 0.006 \\
\hline Presence of DCIS & $0.800(0.298-2.146)$ & 0.658 & - & - \\
\hline Luminal A vs. TNBC & $0.609(0.110-3.362)$ & 0.570 & - & - \\
\hline Luminal B vs. TNBC & $0.880(0.219-3.533)$ & 0.857 & - & - \\
\hline $\mathrm{HER}^{+}$vs. TNBC & $1.016(0.328-3.145)$ & 0.979 & - & - \\
\hline Age & $1.025(0.980-1.072)$ & 0.284 & - & - \\
\hline Marker depth & $0.924(0.825-1.034)$ & 0.170 & - & - \\
\hline Hypoechoic vs. isoechoic & $1.500(0.587-3.833)$ & 0.397 & - & - \\
\hline
\end{tabular}

US, ultrasonography; OR, odds ratio; Cl, confidence interval; DCIS, ductal carcinoma in situ; TNBC, triple-negative breast cancer; HER2, human epidermal growth factor receptor 2.

lesions ( $P=0.022)$. The proportion of US-guided localizations was significantly higher with LigaClip or Cormark markers than with UltraClip markers ( $\mathrm{P}=0.003$ and $\mathrm{P}=0.012$, respectively) and in cases with US-visible residual lesions ( $\mathrm{P}=0.002)$.

In the multivariate analysis, the visibility of LigaClip or Cormark markers was better than that of UltraClip markers ( $P=0.004$ and $P=0.036$, respectively), but the visibility of residual lesion on US did not significantly affect the visibility of the markers $(P=0.062)$. The proportion of US-guided localizations was significantly higher when using LigaClip markers than when using UltraClip markers $(P=0.007)$, but not when using Cormark markers in comparison to UltraClip markers ( $P=0.088)$. Additionally, the presence of a visible residual lesion on US favored US-guided localization ( $\mathrm{P}=0.006$ ).

\section{Discussion}

NAC is an internationally recognized treatment option for breast cancer. The effect of NAC is dramatic in some patients, and the proportion of patients achieving $\mathrm{pCR}$ has been reported to be as high as $32.9 \%$ in some studies [10]. However, an excellent response to NAC can cause difficulties in localization because residual lesions are not clearly visible on images [3,11-13]. Dash et al. [11] reported that no residual tumors were visible on $35.7 \%$ of mammograms (10 of 28). Furthermore, Edeiken et al. [3] reported that $46.9 \%$ of lesions (23 of 49) were not visualized on US or mammography after NAC. In such cases, localization is almost impossible without a breast tissue marker. As localization is needed to decide the extent of surgery and to help the pathologist identify the main lesion, the implantation of breast tissue markers is important for breast cancer patients who undergo NAC.
The visibility of the breast tissue marker is important for localization. US, mammography, and magnetic resonance imaging (MRI) can all be used for localization, and almost all markers are visible on mammography and MRI. However, radiation hazards need to be considered with regard to mammogram-guided localization, and MRI-guided localization is difficult to perform due to excessive time and cost. Thus, US is the most feasible method for localization, but breast tissue markers have relatively inferior visibility on US compared to their visibility on mammography and MRI. In our study, $28.6 \%$ of the markers (12 of 42 ) were not seen on US when no residual tumor was visible on US. This kind of non-visibility of breast tissue markers poses a clinical challenge.

As far as we know, very few studies have compared the visibility of different types of breast tissue markers [14]. Our study shows that the visibility of LigaClip and Cormark may be superior to that of UltraClip, although the use of surgical clips has decreased in recent years due to the introduction of commercial breast markers. LigaClip is thicker than other markers, which might be why it is clearly visible on US (Fig. 3). Cormark is a marker in which a metal clip is surrounded by collagen. The surrounding collagen provides extra contrast, allowing better visibility of the marker (Fig. 4). UltraClip is a marker composed of a non-absorbable polyvinyl alcohol (PVA) polymer with a metallic clip. Our results are broadly similar to those reported by Sakamoto et al. [15], who indicated that $60 \%$ of UltraClip markers were visible. The PVA polymer does not help enhance visibility, so fewer than $50 \%$ of UltraClip markers were visible on US when residual lesions were not visible on US in our study (Fig. 5). However, the surrounding collagen helps enhance visibility, so more than $80 \%$ of Cormark markers were visible. Therefore, radiologists should consider the fact that some markers 
may not be visible after NAC if the tumors show a good response to NAC before inserting the marker. In our study, only $34 \%$ of cancers (246 of 717) reached pCR status, and among them, only 42 cases $(27.5 \%, 42$ of 153$)$ were not visible on US after NAC. Because of the relatively small number of cases with no visible residual tumors, our study might not have an immediate clinical impact at this time, but continuing developments and improvements in NAC and targeted therapy may increase the number of $p C R$ cases, so that our findings may gain more clinical importance in the future.

There are several limitations of this study. First, our study was retrospective, so selection bias may have been present. Second, the number of cases with no visible residual tumors was relatively small, although marker visibility was especially important in those cases. Finally, the visibility of the markers was evaluated on the basis of imaging reading reports written by many radiologists, so interobserver variability may have been present.

In conclusion, the type of breast tissue marker affected the visibility of the marker on US, and more importantly, the localization method used in breast cancer patients who underwent NAC. Thus, the type of breast tissue marker should be considered before clip insertion.

ORCID: Ja Ho Koo: https://orcid.org/0000-0002-5017-6640; Eun-Kyung Kim: https://orcid.org/0000-0002-3368-5013; Hee Jung Moon: https://orcid.org/00000002-5643-5885; Jung Hyun Yoon: https://orcid.org/0000-0002-2100-3513; Vivian Youngjean Park: https://orcid.org/0000-0002-5135-4058; Min Jung Kim: https://orcid.org/0000-0003-4949-1237

\section{Author Contributions}

Conceptualization: Koo JH, Kim EK, Moon HJ, Yoon JH, Park VY, Kim MJ. Data acquisition: Koo JH, Kim MJ. Data analysis or interpretation: Koo JH, Kim MJ. Drafting of the manuscript: Koo JH, Kim MJ. Critical revision of the manuscript: Koo JH, Kim EK, Moon HJ, Yoon JH, Park VY, Kim MJ. Approval of the final version of the manuscript: all authors.

\section{Conflict of Interest}

No potential conflict of interest relevant to this article was reported.

\section{References}

1. Kaufmann M, von Minckwitz G, Mamounas EP, Cameron D, Carey $L A$, Cristofanilli $M$, et al. Recommendations from an international consensus conference on the current status and future of neoadjuvant systemic therapy in primary breast cancer. Ann Surg Oncol 2012;19:1508-1516.

2. Kaufmann M, Hortobagyi GN, Goldhirsch A, Scholl S, Makris A,
Valagussa $\mathrm{P}$, et al. Recommendations from an international expert panel on the use of neoadjuvant (primary) systemic treatment of operable breast cancer: an update. J Clin Oncol 2006;24:1940-1949.

3. Edeiken BS, Fornage BD, Bedi DG, Singletary SE, Ibrahim NK, Strom $E A$, et al. US-guided implantation of metallic markers for permanent localization of the tumor bed in patients with breast cancer who undergo preoperative chemotherapy. Radiology 1999;213:895-900.

4. Phillips SW, Gabriel H, Comstock CE, Venta LA. Sonographically guided metallic clip placement after core needle biopsy of the breast. AJR Am J Roentgenol 2000;175:1353-1355.

5. Shetty MK. Presurgical localization of breast abnormalities: an overview and analysis of 202 cases. Indian J Surg Oncol 2010;1:278-283.

6. Oh JL, Nguyen G, Whitman GJ, Hunt KK, Yu TK, Woodward WA, et al. Placement of radiopaque clips for tumor localization in patients undergoing neoadjuvant chemotherapy and breast conservation therapy. Cancer 2007;110:2420-2427.

7. Fajardo $L L$, Bird RE, Herman $C R$, DeAngelis GA. Placement of endovascular embolization microcoils to localize the site of breast lesions removed at stereotactic core biopsy. Radiology 1998;206:275-278.

8. Thomassin-Naggara I, Lalonde L, David J, Darai E, Uzan S, Trop I. A plea for the biopsy marker: how, why and why not clipping after breast biopsy? Breast Cancer Res Treat 2012;132:881-893.

9. Youn I, Choi SH, Kook SH, Choi YJ, Park CH, Park YL, et al. Ultrasonography-guided surgical clip placement for tumor localization in patients undergoing neoadjuvant chemotherapy for breast cancer. J Breast Cancer 2015;18:44-49.

10. Abdel-Razeq H, Marei L. Current neoadjuvant treatment options for HER2-positive breast cancer. Biologics 2011;5:87-94.

11. Dash N, Chafin SH, Johnson RR, Contractor FM. Usefulness of tissue marker clips in patients undergoing neoadjuvant chemotherapy for breast cancer. AJR Am J Roentgenol 1999;173:911-917.

12. Baron LF, Baron PL, Ackerman SJ, Durden DD, Pope TL Jr. Sonographically guided clip placement facilitates localization of breast cancer after neoadjuvant chemotherapy. AJR Am J Roentgenol 2000;174:539-540.

13. Kim SY, Moon HJ, Kim MJ, Lee SH, Kim EK. US-guided clip implantation for tumor localization in breast cancer patients who undergo neoadjuvant chemotherapy: feasibility study. J Korean Soc Ultrasound Med 2013;32:51-58.

14. Pinkney DM, Shah BA. Prospective comparative study to evaluate the sonographic visibility of five commercially available breast biopsy markers. J Diagn Med Sonogr 2013;29:151-158.

15. Sakamoto N, Ogawa Y, Tsunoda Y, Fukuma E. Evaluation of the sonographic visibility and sonographic appearance of the breast biopsy marker (UltraClip ${ }^{\circledR}$ ) placed in phantoms and patients. Breast Cancer 2017;24:585-592. 